\title{
FRACTIONAL ORDER BASED COMPUTED TORQUE CONTROL OF 2-LINK ROBOTIC ARM
}

\author{
Haris Anwaar', Yin Yixin', Salman ljaz², Muhammad Ammar Ashraf', Waqas Anwaar ${ }^{3}$ \\ 1 University of Science and Technology, 30 Xueyuan Road, Haidian District, Beijing 100083, P. R. China, e-mails: \\ harisishere@hotmail.com, yyx@ies.ustb.edu.cn, ch.ammar.ashraf@gmail.com \\ 2 Beihang University, No. 37 Xueyuan Road, Haidian District, Beijing 100083, P. R. China, e-mail: \\ salman_ijaz830@hotmail.com \\ 3 Comsats Institute of Information Technology, Park Road, Tarlai Kalan, Islamabad, 45550, Pakistan, e-mail: \\ waqasanwaar@hotmail.com
}

Received: 2017.12.01

Accepted: 2018.02.01

Published: 2018.03.01

\begin{abstract}
The paper proposes the application of fractional order controller in position tracking control of 2-link nonlinear robotic arm. The nonlinear system dynamics is linearized using inverse dynamics of the model and fractional order PID controller is designed to deal with remaining tracking errors. The optimal values of controller parameters are calculated using Nelder-Mead optimization technique based on desired design criteria. The objective function is designed using weighted sum approach on each performance specification based on transient domain parameters. It can be seen from simulation results that fractional order controller together with computed torque controller improved tracking performance of proposed system as compared to PID controller used in the outer loop. Moreover, the robustness of proposed scheme is checked by applying the disturbance signal at control input channels of 2-link nonlinear robotic arm links.
\end{abstract}

Keywords: fractional order PID controller; computed torque controller; 2-link robotic arm; Nelder-Mead optimization

\section{INTRODUCTION}

Robotic arms are widely used in various industries like process industries, medical fields, nuclear plants to name a few [1] to perform several tasks like picking and placing, paint spraying, welding, material deposition for 3D printing, calligraphy and so on. All such applications require the end effector of the robotic arm to achieve precise level of positioning, faster response and robust behavior in the presence of external disturbance and varying load conditions. There are coupled nonlinearities (friction, backlash due to mechanical transmission) and time-varying dynamics in robotic manipulator system that can cause big tracking error especially when fast position tracking is required. Moreover, the pa- rameters of both mechanical parts and actuating systems have uncertainty, which also contributes in its complexity. Therefore, the control of robotic arm has always been the research area because of its complex nature.

Many control algorithms have been designed to address these issues such as optimal control [2], model predictive control [3], robust neural control [4], neural network control with state and output feedback [5], neural network based robust $H_{\infty}$ controller for manipulators with uncertainties [6], robust fuzzy controller with parametric uncertainties and external disturbances [7], adaptive fuzzy sliding mode controller has been designed for different burden conditions and in the presence of dynamic uncertainties[8], variable structure control i.e., sliding mode control [9], com- 
puted torque control (CTC) with fuzzy controller [10], and so on. Although many nonlinear control methods exist for robotic manipulators position control but PID controller still gets wide acceptance because of ease of design, simplicity of control structure, and variety of tuning schemes, and also better performance in the presence of external disturbances and model uncertainties i.e., fuzzy PID controller designed for a manipulator in case of uncertainties [11], particle swarm optimization tuned-PID controller [12] to name a few. PID controllers find an extensive use in other industries too. A survey was carried out by Japan Electric Measuring Instrument Manufacturer's Association in 1989 for the state of process control systems [13], and it was found out that more than 90 percent of the control loops were PID type. Although PID controllers are good choice for robotic manipulators as introduced above, but they have limitations, i.e., they are good in slow motions, controller performance degrades in case of high speed requirements [10].

The above-mentioned control strategies applied to the complex and practical control problem of robotic manipulator show worthwhile results. But the traditional schemes cannot be ignored, among them computer torque control shortly named as CTC [14] is widely used scheme for the robotic system due to its easy design and good performance. CTC requires the perfect model of the robotic manipulator to linearize [10] or decouple the system dynamics so that each joint can be controlled individually using well-recognized control strategies. It seems that CTC control is sometimes applied, unless that in the scheme proposed by [14], without a feedback to compensate model errors; but a perfect model including all friction effects is never possible in practice, therefore all CTC methods have a closed loop correction, not only the one proposed in [14] and [15].

In recent years, fractional order modeling and control of nonlinear systems have got great attention in control system applications. Fractional order PID (FOPID) controller has been used extensively in control of nonlinear systems. The availability of more adjustable parameters theoretically makes FOPID controller more effective as compared to the classical PID controller. Better robustness is achieved in terms of system parameters, controller parameters and external load conditions using FOPID controller. It is thus suitable for robotic arm position control problem due to the inherent nonlinearities and mechanical linkage in these that lead the output undergoing large settling time and steady- state error. This paper proposes a hybrid strategy that combines fractional order controller with traditional CTC that not only improve the dynamical behavior of system subject to the disturbance and other modeling error but also achieve the precise tracking of end effector of the robotic manipulator.

To justify the dominant performance of FOPID controller as compared to PID controller, a brief survey is carried out. In case of SISO systems, FOPID has been applied for the DC motor speed control in [16], controlling the bioreactor system [17], backlash vibration suppression control for the torsional system [18], and electro-hydraulic servo system (EHSS) position tracking control [19]. For MIMO systems, FOPID controller has also been successfully applied in $[20,21,22]$. It can be seen from these applications that performance of controller entirely depends on finetuning of controller parameter. The tuning of the parameters depends upon the optimization technique, fitness function and search space dimension. So an appropriate tuning algorithm is required to be chosen to find the most suitable parameters. There are numerous tuning algorithms, which differ in terms of convergence speed. In [20], Nelder-mead is shown better than PSO as it is a geometric algorithm and provides faster convergence. So, this method is chosen in this paper.

In robotic manipulator systems, fractional order controller has been applied to hexapod robot having viscous friction and flexibility at the joints in the legs in [23]. The controller is validated experimentally and it was concluded that fractionalorder $P D^{\alpha}$ controller gives better robustness as compared to an integer-order PD algorithm. Not only the $P D^{\alpha}$ scheme is possible instead of PD, but other fractional order controls are also possible, such as the $P D^{1 / 2}$ controller designed in [24]. FOPID controller is designed in [25] for robotic manipulator based on the linear time invariant model of system and the parameters are tuned using optimization techniques. Simulation results show better performance than integer order PID controller. Moreover, in [26, 27, 28] fractional PID controller with output feedback has been used for robotic manipulators and their robustness has been verified.

The main contribution of this paper is the design of novel control strategy to improve the tracking performance of robotic manipulator in which CTC together with fractional order PID controller is designed in two loops. The first loop is an inner loop that consists of inverse dynamics 
of the robotic arm in feed forward path with the system. The second one is an outer loop, which is optimally tuned fractional order PID controller and its performance is compared with integer order PID controller. The nonlinear dynamics of system is formulated using Lagrange-Euler method. Moreover, the parameters of both controllers (CTC-FOPID and CTC-PID) are tuned using Nelder-Mead optimization technique based on the desired performance index and their performances are compared in the end based on transient domain parameters.

The rest of paper has been organized in following way. In section 2, robotic model and its nonlinear state-space model and dynamic model are presented. Section 3 describes the detailed control strategy. In section 4, the optimization technique used to tune the five parameters of fractional-order PID controller is described. In section 5, FOPID controller design parameters and performance parameters are given and in the end, simulation results are shown. Finally, in section 6 conclusion is given.

\section{PRELIMINARIES}

\section{Stability Condition of Fractional order System}

The BIBO (bounded input-bounded output) stability criteria for a commensurate-order transfer function $T_{f}(s)$ is given by Matignon's stability theorem described as under:

Theorem 1 (Matignon's stability theorem) [29]: Fractional order transfer function $T_{f}(s)=\frac{N(s)}{D(s)}$ is said to be stable if and only if the given condition is satisfied in $\rho$-plane:

$$
|\arg (\rho)|>q \frac{\pi}{2} \text {, here } \rho=s^{q},(0<q<1) \text { with }
$$

$\forall \rho \in C, D(\rho)=0$, where $q$ represents fractional commensurate order.

When $\sigma=0$ is a single root of $D(\rho)$, the system can't be stable. Fig. 1 represents the condition in the graphical form

\section{NONLINEAR MODEL OF ROBOTIC ARM}

The industrial robot used in this paper is 2-R planar robot mentioned in [30] and its nonlinear model is given in equation (1). Dynamics of used robotic arm is shown in Fig. 2 below [31]. $\left[\begin{array}{ll}z_{11} & z_{12} \\ z_{21} & z_{22}\end{array}\right]\left[\begin{array}{l}\ddot{\theta}_{1} \\ \ddot{\theta}_{2}\end{array}\right]+\left[\begin{array}{l}B_{11} \\ B_{21}\end{array}\right]+\left[\begin{array}{l}V_{11} \\ V_{22}\end{array}\right]+\left[\begin{array}{l}g_{11} \\ g_{22}\end{array}\right]=\left[\begin{array}{l}\tau_{1} \\ \tau_{2}\end{array}\right]$

Here

$$
\begin{aligned}
& z_{11}=I_{11}+I_{22}+m_{1} l_{c 1}^{2}+m_{2} l_{1}^{2}+m_{2} l_{c 2}^{2}+ \\
& +2 m_{2} l_{1} l_{c 2} \cos \theta_{2}+m_{0} l_{1}^{2}+m_{0} l_{2}^{2}+2 m_{0} l_{1} l_{c 2} \cos \theta_{2} \\
& z_{12}=I_{22}+m_{2} l_{c 2}^{2}+m_{2} l_{1} l_{c 2} \cos \theta_{2}+m_{0} l_{2}^{2}+ \\
& +m_{0} l_{1} l_{c 2} \cos \theta_{2} \\
& z_{21}=z_{12} \\
& z_{22}=I_{22}+m_{2} l_{c 2}^{2}+m_{0} l_{2}^{2} \\
& B_{11}=-m_{2} l_{1} l_{c 2}\left(2 \dot{\theta}_{1}+\dot{\theta}_{2}\right) \dot{\theta}_{2} \sin \theta_{2}-m_{0} l_{1} l_{2} \\
& \left(2 \dot{\theta}_{1}+\dot{\theta}_{2}\right) \dot{\theta}_{2} \sin \theta_{2} \\
& B_{21}=m_{2} l_{1} \dot{\theta}_{1}^{2} l_{c 2} \sin \theta_{2}+m_{0} l_{1} \dot{\theta}_{1}^{2} l_{2} \sin \theta_{2} \\
& V_{11}=b_{11} \dot{\theta}_{1} \\
& V_{22}=b_{21} \dot{\theta}_{2} \\
& g_{11}=m_{1} l_{c 1} g \cos \left(\theta_{1}\right)+m_{2} g\left(l_{c 2} \cos \left(\theta_{1}+\theta_{2}\right)+\right. \\
& \left.+l_{1} \cos \left(\theta_{1}\right)\right)+m_{0} g\left(l_{2} \cos \left(\theta_{1}+\theta_{2}\right)+l_{1} \cos \left(\theta_{1}\right)\right) \\
& g_{22}=m_{2} l_{c 2} g \cos \left(\theta_{1}+\theta_{2}\right)+m_{0} l_{2} g \cos \left(\theta_{1}+\theta_{2}\right)
\end{aligned}
$$

Here $\theta^{1}$ and $\theta^{2}$ represent the positions for link1 and link-2,

$\tau_{1}$ and $\tau_{2}$ represent torques on link- 1 and link-2,

$l_{1}$ and $l_{2}$ represent the link-1 and link-2 lengths,

$I_{11}$ and $I_{22}$ represent lengthwise centroid inertia,

$l_{C 1}$ and $l_{c 2}$ represent the distance from the joints of the links to their center of gravity, $b_{11}$ and $b_{21}$ are the coefficients of friction at joints,

$m_{1}$ and $m_{2}$ represent the masses of link-1 and link-2 and

$m_{0}$ represents the mass on the tip of the arm.

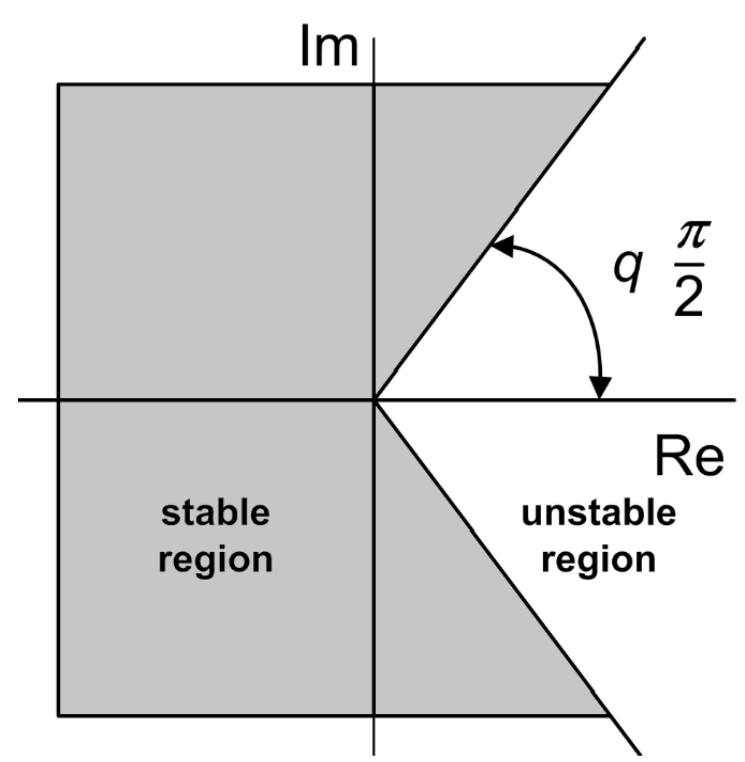

Fig. 1. Stability region for LTI fractional order system with order $0<\mathrm{q}<1$ 


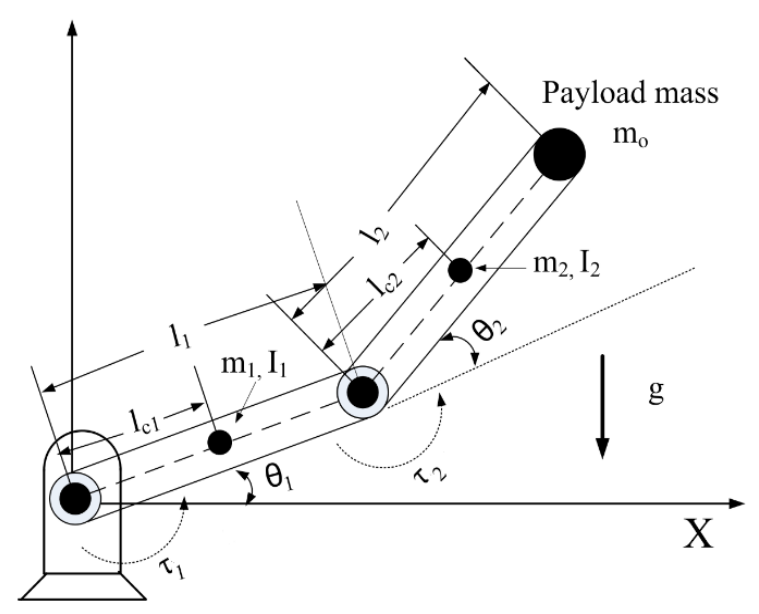

Fig. 2. 2-R planar robot with payload at the tip

The physical parameters of the robotic arm are shown in Table 1 below.

Considering the system model mentioned in equation (1), the mass matrix can be extracted as

$$
M=\left[\begin{array}{ll}
z_{11} & z_{12} \\
z_{21} & z_{22}
\end{array}\right]
$$

Defining a vector of generalized coordinates:

$$
\mathrm{q}=\left[\begin{array}{l}
\theta_{1} \\
\theta_{2}
\end{array}\right]
$$

leads to a second-order nonlinear differential equation

$$
\ddot{q}=\frac{1}{\mathrm{M}} *\left(-\left[\begin{array}{l}
B_{11} \\
B_{21}
\end{array}\right]-\left[\begin{array}{l}
V_{11} \\
V_{22}
\end{array}\right]-\left[\begin{array}{l}
g_{11} \\
g_{22}
\end{array}\right]+\left[\begin{array}{l}
\tau_{1} \\
\tau_{2}
\end{array}\right]\right)
$$

present link-1 and link-2 positions. The respective time derivatives of link-1 and link-2 i.e., $\dot{\theta}_{1}$ and $\dot{\theta}_{2}$ are the angular velocities of the link-1 and link-2 of the robotic arm. The output signals are

\begin{tabular}{|c|c|c|}
\hline Parameters & Link 1 & Link 2 \\
\hline Mass & $0.39292 \mathrm{~kg}$ & $0.09440 \mathrm{~kg}$ \\
\hline $\begin{array}{l}\text { Acceleration due to gravity } \\
\text { (g) }\end{array}$ & $\begin{array}{l}9.81 \\
\mathrm{~m} / \mathrm{s}^{2}\end{array}$ & $\begin{array}{l}9.81 \\
\mathrm{~m} / \mathrm{s}^{2}\end{array}$ \\
\hline Length & $0.2032 \mathrm{~m}$ & $0.1524 \mathrm{~m}$ \\
\hline $\begin{array}{l}\text { Lengthwise centroid inertia } \\
\text { of link }\end{array}$ & $\begin{array}{l}0.001141 \\
\mathrm{~kg} \cdot \mathrm{m}^{2}\end{array}$ & $\begin{array}{c}0.002024 \\
\mathrm{~kg} \cdot \mathrm{m}^{2}\end{array}$ \\
\hline Friction value at joints & $\begin{array}{c}0.14123 \\
\mathrm{~N}-\mathrm{m} / \text { radian/s }\end{array}$ & $\begin{array}{c}0.353077 \\
\mathrm{~N}-\mathrm{m} / \text { radian/s }\end{array}$ \\
\hline $\begin{array}{l}\text { Distance from the joint of } \\
\text { link to its center of gravity }\end{array}$ & $0.10464 \mathrm{~m}$ & $0.08178 \mathrm{~m}$ \\
\hline
\end{tabular}
$\theta_{1}$ and $\theta_{2}$ and the following nonlinear state equa-

Table 1. Model parameters of 2-link robotic arm [31] tions of 2-link robotic arm are derived from the model given in [30]

$$
\frac{d}{d t}\left(\theta^{\prime}\right)=\left[\begin{array}{c}
\left(\frac{1}{z_{11}}\right)\left(\tau_{1}-B_{11}-g_{11}-V_{11}\right)-\left(\frac{z_{12}}{\left(z_{11} Z\right.}\right) \\
\left(\tau_{2}-B_{21}-g_{22}-V_{22}-\bar{z}\right) \\
\theta_{2} \\
\frac{1}{z}\left(\tau_{2}-B_{21}-g_{22}-V_{22}-\bar{z}\right)
\end{array}\right]=\left[\begin{array}{c}
\theta_{1} \\
\dot{\theta}_{1} \\
\theta_{2} \\
\dot{\theta}_{2}
\end{array}\right]
$$

here

$$
z=z_{11}-\frac{z_{21} z_{12}}{z_{11}}
$$

and

$$
\bar{z}=\left(\frac{z_{21}}{z_{11}}\right)\left(\tau_{1}-B_{11}-g_{11}-V_{11}\right)
$$

\section{CONTROL SCHEME}

In this paper, the proposed control design comprises of two loops. The inner loop is inverse dynamics loop in feedforward path to linearize the model and the outer loop contains FOPID controller which removes the remaining modeling errors and uncertainties. The block diagram representation of overall control strategy is shown in Fig. 3. The proposed technique is compared with an already existing technique that used PID controller in the outer loop with CTC to achieve the desired performance. The proposed control system is designed with the consideration of the improvement in the transient domain parameters, increase in the range of operating frequency and disturbance rejection ability. Since the FOPID controller has five tuning parameters that are two additional parameters i.e., Derivative fractional power $(\mu)$ and Integral fractional power $(\lambda)$ as compared to conventional PID controller so it provides more adjustable time and frequency responses allowing fulfillment of better as well as robust performance. Moreover, in order to achieve full benefits of FOPID controller, optimal tuning of controller parameters according to well design performance criteria is necessary. In this paper, Nelder-Mead optimization [32] technique is employed to tune both PID and FOPID controller. In this work, a design criterion is formulated using weighted sum approach that includes transient domain performance specifications i.e. overshoot $(\sigma e)$, settling time $\left(T_{s}\right)$ and integrated absolute error $(I A E)$. The performance criteria $P(s)$ is considered by allocating the weights to above given factors as 


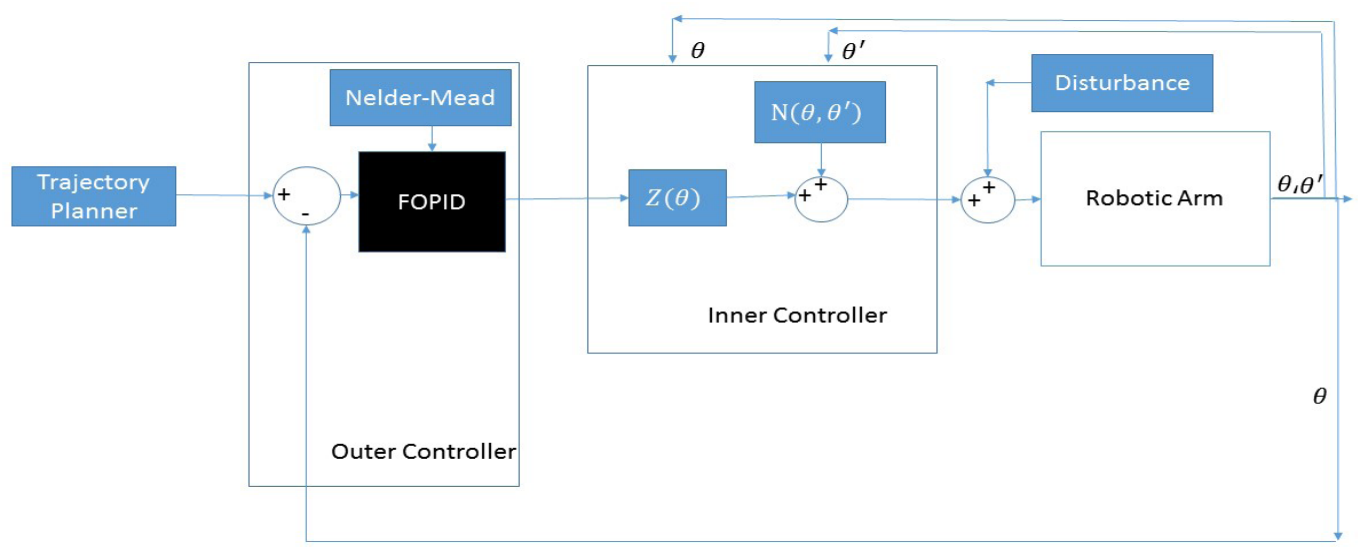

Fig. 3. Computed Torque FOPID control design strategy for 2-link robotic arm

$$
P(s)=\omega_{1} \sigma_{e}+\omega_{2} T_{s}+\omega_{3} \int I A E d t
$$

In Fig. 3, $\mathrm{N}\left(\theta, \theta^{\prime}\right)=\mathrm{B}\left(\theta, \theta^{\prime}\right)+\mathrm{g}(\theta)+\mathrm{V}\left(\theta^{\prime}\right)$.

\section{Computed Torque Controller}

CTC belongs to inverse dynamic control class of controllers. The principle of this controller is that inverse dynamics of the nonlinear system is cascaded with the system itself such that the overall system approaches a unity gain. The inverse of the nonlinear system is not perfect in practice so, in order to compensate the remaining modeling errors and reduce the effect of external disturbance, an output feedback loop is required. Hence, it can be said that CTC has a PD/PID controller in the outer loop plus a feedback linearizing controller in the internal loop. In this work, in comparison to conventional CTC, the outer PID controller has been replaced by fractional order PID controller. This replacement provides additional robustness and inhibits errors more efficiently. The new input control laws are expressed by the following equations:

$$
\begin{gathered}
{\left[\begin{array}{l}
\tau_{1} \\
\tau_{2}
\end{array}\right]=\left[\begin{array}{l}
B_{11} \\
B_{21}
\end{array}\right]+\left[\begin{array}{l}
V_{11} \\
V_{22}
\end{array}\right]+\left[\begin{array}{l}
g_{11} \\
g_{22}
\end{array}\right]+\left[\begin{array}{l}
u_{1} \\
u_{2}
\end{array}\right]} \\
{\left[\begin{array}{l}
u_{1} \\
u_{2}
\end{array}\right]=M *\left[\begin{array}{l}
v_{1} \\
v_{2}
\end{array}\right]} \\
v_{1}=\ddot{\theta}_{1 d}+K_{d_{1}} s^{\mu_{1}} *\left(\dot{\theta}_{1 \mathrm{~d}}-\dot{\theta}_{1}\right)+ \\
+K_{p_{1}} *\left(\theta_{1 \mathrm{~d}}-\theta_{1}\right)++K_{i_{1}} s^{-\lambda_{1}} \int\left(\theta_{1 \mathrm{~d}}-\theta_{1}\right) d t
\end{gathered}
$$

$$
\begin{gathered}
v_{2}=\ddot{\theta}_{2 d}+K_{d_{2}} s^{\mu_{2}} *\left(\dot{\theta}_{2 \mathrm{~d}}-\dot{\theta}_{2}\right)++ \\
+K_{p_{2}} *\left(\theta_{2 \mathrm{~d}}-\theta_{2}\right)++K_{i_{2}} s^{-\lambda_{2}} \int\left(\theta_{2 \mathrm{~d}}-\theta_{2}\right) d t
\end{gathered}
$$

Here $K_{p 1}, K_{d 1}$ and $K_{i 1}$ are proportional, derivative and integral gains for the position errors of link-1 and $\mu_{1}$ and $\lambda_{1}$ represent the fractional power of the derivative and integral for link-1 error terms. Similarly, $\mathrm{K}_{\mathrm{p} 2}, \mathrm{~K}_{\mathrm{d} 2}$ and $\mathrm{K}_{\mathrm{i} 2}$ represent proportional, derivative and integral gains for the position errors of link-2 and $\mu_{2}$ and $\lambda_{2}$ represent the fractional power of the derivative and integral for link-2 error terms respectively. Subscript d represents the desired value in equation (11) and equation (12).

\section{Fractional order PID controller}

The advancement in the computational power made the fractional order modeling and fractional order control systems implementation much easier. Fractional order control can achieve the clear-cut design of robust control systems. In the future, fractional order models and controllers are expected to take place of integer order controllers in industries, and other areas due to the advancements in dealing of the computational complexity and methods and tools developed to approximate the solutions of fractional calculus.

Oustaloup (1991) was the first to use FOC to develop as the crone controller. FOPID controller is represented in transfer function form as

$$
\begin{gathered}
C(s)=K_{P}+K_{d} s^{\mu}+K_{i} s^{-\lambda} \\
\text { where } \mu, \quad \lambda>0
\end{gathered}
$$


From equation (9), it can be seen that FOPID controller as compared to simple PID controller (three controllable parameters i.e., Proportional gain $\left(K_{p}\right)$, Derivative gain $\left(K_{d}\right)$, Integral gain $\left(K_{i}\right)$ has two more controllable parameters i.e., Derivative fractional power $(\mu)$ and Integral fractional power $(\lambda)$. It can be easily observed that due to the advent of two more parameters i.e., $\mu$ and $\lambda$ numerous choices are available for the development of controller (continuously defined on the $(\mu-\lambda)$-plane) shown in Table 2. FOPID controller is represented in block diagram in Fig. 4

While applying FOPID controller, two problems are critical. One is approximation of the model or model realization and the other is the tuning of the controller parameters. Model approximation mainly involves two techniques. One of them is interpolation technique and the other one is curve fitting such as Oustaloup filter [33]. The tuning problem of the controller parameters is actually more critical than the first one. Controller parameters tuning can be termed as an optimization process for which controller response form, setpoint and process variable error can be termed as the performance criteria [19]. Normally, the tuning of PID parameters involves the set-point following problem or the load disturbance rejection task, whereas in some circumstances both of them have the key significance. While tuning, the control effort is also of crucial importance as it defines the final cost of the product and the wear and life-span of the actuator. Therefore, control effort

Table 2. Controller types based on $\lambda$ and $\mu$

\begin{tabular}{|c|c|}
\hline Fraction Value & Type of Controller \\
\hline$\lambda=0, \mu=0$ & $\mathrm{P}$ \\
\hline$\lambda=0, \mu=1$ & $\mathrm{PD}$ \\
\hline$\lambda=1, \mu=0$ & $\mathrm{PI}$ \\
\hline$\lambda=1, \mu=1$ & $\mathrm{PID}$ \\
\hline
\end{tabular}

is highly desirable to be kept at a minimum level. Moreover, the issue of robustness also needs to be taken into account [34]. Hence, some efficient optimization technique is highly desirable which can give the most accurate tuning parameters in accordance with the desired specification.

In this paper, to control the position of the 2-link robotic arm, a FOPID controller is designed. The FOPID controller parameters tuning is done using Nelder-Mead (N-M) optimization algorithm. Based on the criteria given in (6), the fitness function is designed.

\section{OPTIMIZATION TECHNIQUE FOR PARAMETER TUNING}

This section explains briefly the optimization algorithm used in this paper i.e., Nelder-Mead algorithm. This optimization algorithm is used in this paper for the tuning of the parameters of FOPID and PID controllers. Based on the performance index defined in equation (6), the objective function is minimized such that closed-loop system is internally stable.

Nelder-Mead tuning is chosen for the robotic arm because it provides faster convergence as compared to other optimization techniques i.e., PSO technique [20].

\section{Nelder-Mead (N-M) optimization algorithm}

Nelder-Mead optimization [35] method belongs to the general class of direct search methods. N-M optimization finds the local minima using a simplex-based algorithm. This method is well recognized for parameter optimization because of computation simplicity. This method resembles closely to particle swarm optimization (PSO) and differential evolution (DE) [36]. The

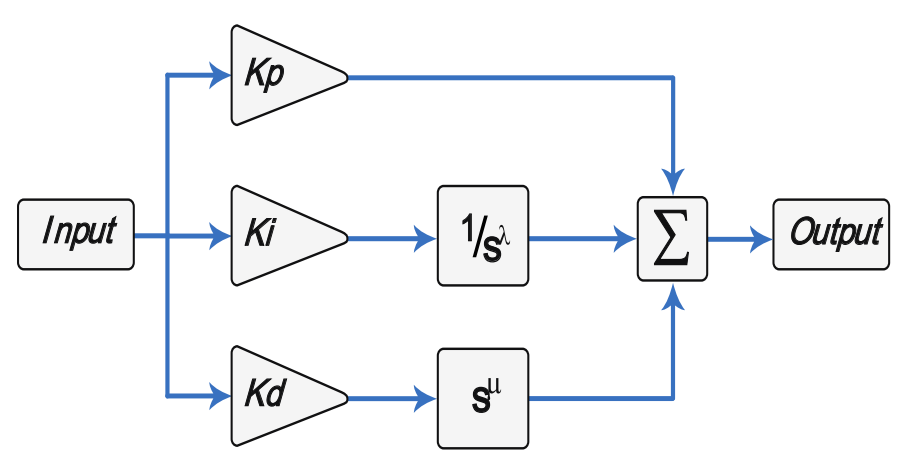

Fig. 4. Block diagram representation of FOPID controller 
pattern search approach is applied in this method using $n+1$ dimensional shape.

Moreover, for this method the derivative information is not required to minimize the function of $n$ variables, making it suitable for nonsmooth function problems. The search done using Nelder-Mead algorithm is a geometric operation which includes replacing one vertex of simplex by its reflected image, expanding, contracting, and shrinking of the simplex. Nelder-Mead algorithm requires a continuous search space same way as the original versions of DE and PSO, and the points in space are represented as vectors of real numbers [37]. Nelder-Mead technique has already been applied for the tuning of the PID controller parameters applied for the position tracking control of EHSS [38],[20]. Better and fast tracking performance is achieved as seen from the simulation results in comparison to selftuning fuzzy approach. Fig. 5 represents the block diagram representation of for N-M search in a compact way.

In this paper, FOPID controller $K_{p}, K_{i}, K_{d,}$ $\mu$ and $\lambda$ parameters are tuned using the NelderMead optimization technique. The tool is discussed in detail in [39] for the identification of these parameters using N-M technique. The fitness function is given by performance criteria provided in equation (6).

\section{FOPID CONTROLLER DESIGN AND NONLINEAR MODEL SIMULATIONS}

This section has been divided into two parts. In the first part the design parameters and performance parameters of FOPID controllers are given and in the second part, simulation results are given for the tracking of different reference signals for the 2-link nonlinear robotic arm.

\section{FOPID controller design}

The FOPID controller is designed to achieve precise tracking control of 2-link robotic manipulator as it is shown in block diagram in Fig. 3. In order to tune the 5-dimensional search space, i.e. 5 FOPID parameters, Nelder-Mead optimization algorithm is designed to satisfy the constraints as follows: Settling time is set to $2 \mathrm{~s}$, rise time to $1 \mathrm{~s}$, percentage overshoot of more than $10 \%$ is not allowed and steady-state error is set below 0.1 s respectively. In N-M algorithm, $\kappa+1$ dimensional simplex is chosen, where $\kappa=5$ for our case and represents the number of parameters to be used in optimization process. At the start, these FOPID controller parameters are set as $\mathrm{K}_{\mathrm{p}}=\mathrm{K}_{\mathrm{i}}=\mathrm{K}_{\mathrm{d}}=1, \mu=\lambda=0.5$. The values of weight functions mentioned in equation (6) are chosen as $\mathrm{w}_{1}=\mathrm{w}_{2}=0.6, \mathrm{w}_{3}=1$. The controller is analyzed and implemented using FOMCON toolbox designed for MATLAB [40]. It supports the simulation, optimization and realization of FOPID controllers via its built-in functions. The optimized parameter values for PID and FOPID controllers for link-1 and link-2 respectively are shown in Table 3 and 4 . Table 5 and 6 compare the performance parameters of both controllers based on the objective function given in equation (6).

The controller performance is tested by using the optimally tuned parameters in the closed-loop configuration of the system for various reference tracking signals. In the upcoming section, the simulation results are described for different cases.

\section{Stability analysis}

The closed-loop stability of the control scheme can be proved by assuming that CTC

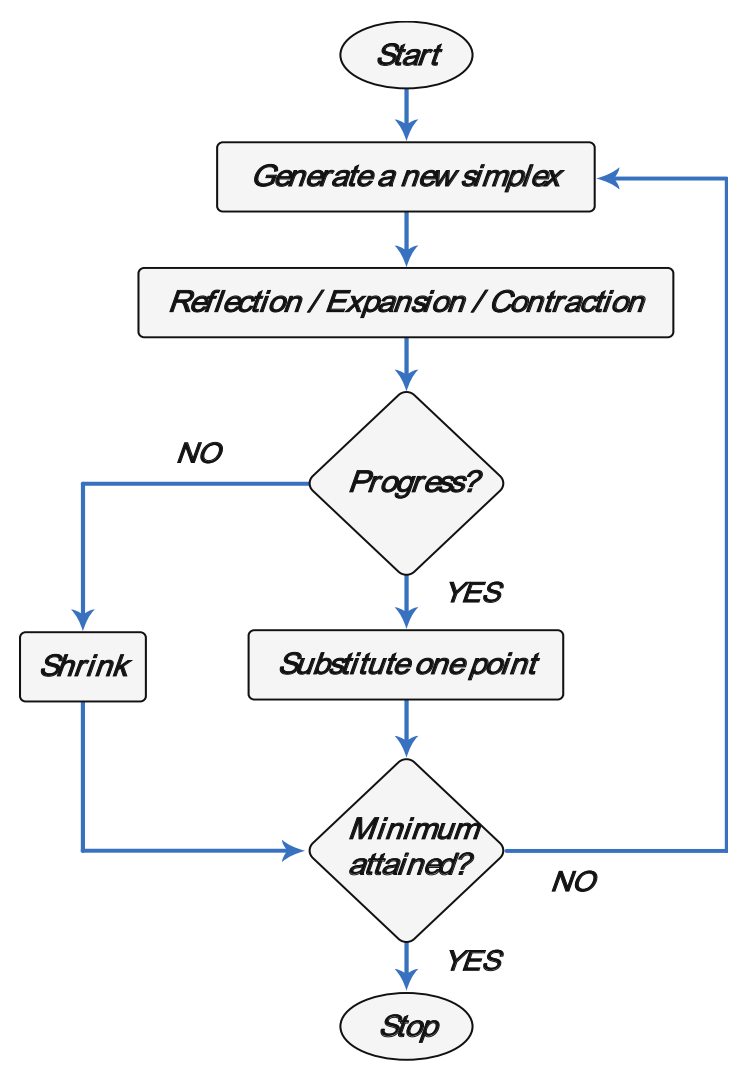

Fig. 5. Algorithm for Nelder-Mead optimization [20] 
Table 3. Parameters obtained for PID and FOPID controller using N-M tuning

\begin{tabular}{|c|c|c|}
\hline Parameters & PID (N-M) & FOPID (N-M) \\
\hline$K_{p}$ & 378.3 & 289.67 \\
\hline$K_{i}$ & 12.38 & 15.1 \\
\hline$K_{d}$ & 213.03 & 318.54 \\
\hline$\lambda$ & - & 0.53 \\
\hline$\mu$ & - & 0.61 \\
\hline
\end{tabular}

Table 4. Parameters obtained for PID and FOPID controller using N-M tuning

\begin{tabular}{|c|c|c|}
\hline Parameters & PID (N-M) & FOPID (N-M) \\
\hline$K_{p}$ & 503.45 & 421.23 \\
\hline$K_{i}$ & 15.84 & 26.93 \\
\hline$K_{d}$ & 434.41 & 467.71 \\
\hline$\lambda$ & - & 0.52 \\
\hline$\mu$ & - & 0.501 \\
\hline
\end{tabular}

Table 5. Performance comparison of PID and FOPID controller for link-1

\begin{tabular}{|c|c|c|}
\hline $\begin{array}{c}\text { Performance } \\
\text { Parameters }\end{array}$ & PID (N-M) & FOPID (N-M) \\
\hline$T_{s}$ & $0.95 \mathrm{~s}$ & $0.2 \mathrm{~s}$ \\
\hline Overshoot & $0 \%$ & $0 \%$ \\
\hline
\end{tabular}

Table 6 Performance comparison of PID and FOPID controller for link-2

\begin{tabular}{|c|c|c|}
\hline $\begin{array}{c}\text { Performance } \\
\text { Parameters }\end{array}$ & PID (N-M) & FOPID (N-M) \\
\hline$T_{s}$ & $2.9 \mathrm{~s}$ & $0.2 \mathrm{~s}$ \\
\hline Overshoot & $11 \%$ & $0 \%$ \\
\hline
\end{tabular}

drives the open loop gain to unity. However, practically there are incomplete cancellations of nonlinear terms (due to imperfectly known parameters or inevitable friction effects). By assuming ideal case, the transfer function of the FOPID controller plays the main role for the closed loop stability of the system. The equivalent open loop transfer function $\mathrm{G}_{\mathrm{p} 1-\mathrm{eq}}$ of plant with CTC controller can be written as under

$$
G_{p_{1-e q}}=G_{p_{1}} G_{p_{1-\text { inverse }}}
$$

where: $G_{p 1}$ represents link-1 plant model and $G_{p 1-\text { inverse }}$ is plant inverse model.

The stability criteria mentioned in the preliminary part is considered to verify the stability.
The closed-loop transfer function is represented by $\mathrm{G}_{\mathrm{p} 1-\text { link } 1}$ in equation (15)

$$
G_{c l-l i n k 1}=\frac{G_{c-l i n k 1} G_{p 1-e q}}{1+G_{c-l i n k 1} G_{p 1-e q}}
$$

where: $G_{c-l i n k 1}=289+\frac{15}{s^{0.53}}+318 s^{0.61}$

which represents the FOPID controller transfer function for link-1. The stability plot for link-1 is shown in Fig.6.

In the same way, for the case of link-2, the equivalent open loop transfer function $\mathrm{G}_{\mathrm{p} 2}$ - eq of plant with CTC controller can be written in the following equation

$$
G_{p_{2-e q}}=G_{p_{2}} G_{p_{2-\text { inverse }}}
$$

where: $G_{p 2}$ and $G_{p 2 \text {-inverse }}$ represent link-2 plant model and plant inverse model respectively.

Now, the closed loop transfer function for link-2 $\mathrm{G}_{\mathrm{cl}-\text { link } 2}$ is given by

$$
G_{c l-l i n k 2}=\frac{G_{c-l i n k 2} G_{p 2-e q}}{1+G_{c-l i n k 2} G_{p 2-e q}}
$$

where: $G_{c l-l i n k 2}=\frac{G_{c-l i n k 2} G_{p 2-e q}}{1+G_{c-l i n k 2} G_{p 2-e q}}$

which represents the FOPID controller transfer function for link-2 and the corresponding stability plot is shown in Fig. 7.

It can be inferred from Fig. 6 and Fig. 7 that both the closed loops are stable with commensurate order 0.1 as no poles exist in the red region as suggested by Matignon's stability criteria described before. Moreover, it can be also seen from [41] that fractional order controller combined with CTC has stable poles in a closed loop. Moreover, the controller with fractional power has more stability region as compared to integer order PID controllers.

\section{Simulation results}

In this section, the simulation results for different cases are shown when the FOPID controller is applied for the position control of the robotic arm. Nelder-Mead optimization technique has been used to optimize the tuning of the parameters for the controllers and in the end, the simulation results for different cases have been compared. In order to test the robust nature of 


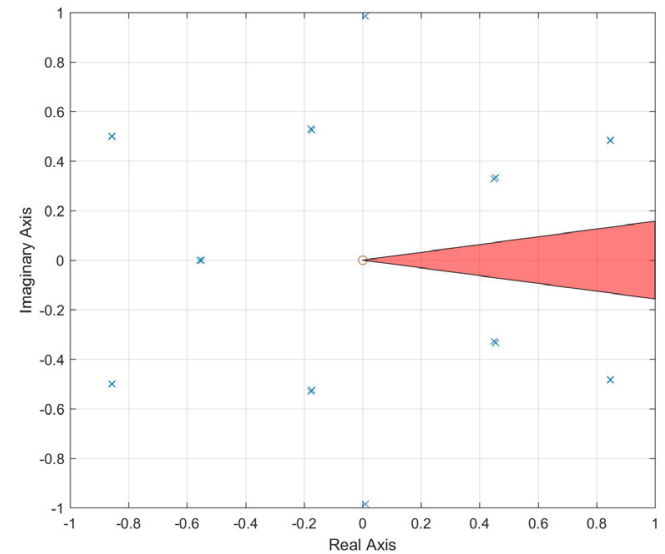

Fig. 6. Stability plot for FOPID controller for link-1

the FOPID controllers designed, disturbance signal has been entered at the control input channel. Moreover, by applying different reference signals the tracking performance and the feasibility of the proposed controllers are tested. The shown simulation results are based on the nonlinear model of the robotic arm and the results are also compared with the conventional PID controller in order to verify the improvement and benefit of the proposed technique. The value of $\mathrm{m}_{0}$ used for the simulation is $0.567 \mathrm{~kg}$.

\section{Tracking Performance under Step Input:}

The tracking performance of the proposed controller in case of step reference input applied at input of both links is shown in Fig. 8 and Fig. 9. The dominant performance of FOPID controller as compared to PID controller can be seen clearly. In Fig. 8, the FOPID controller settles more quickly while PID controller possesses some over-

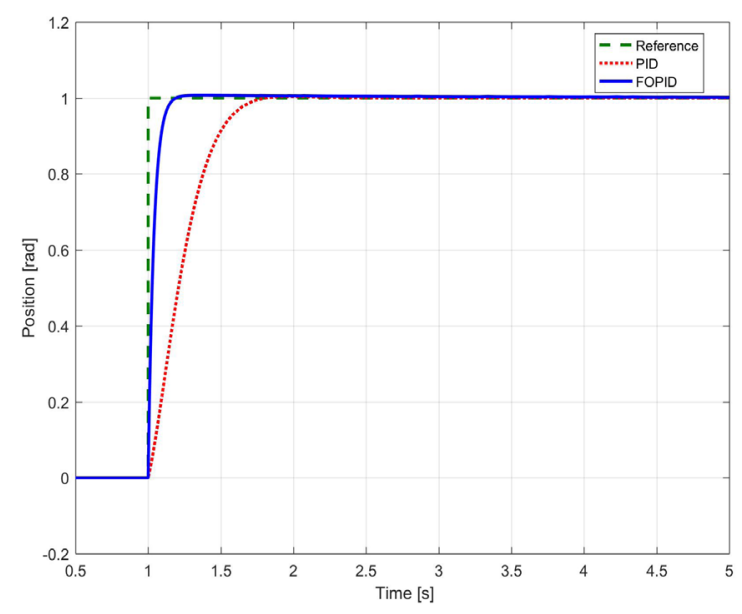

Fig. 8. Tracking performance for robotic arm for link 1 for step input

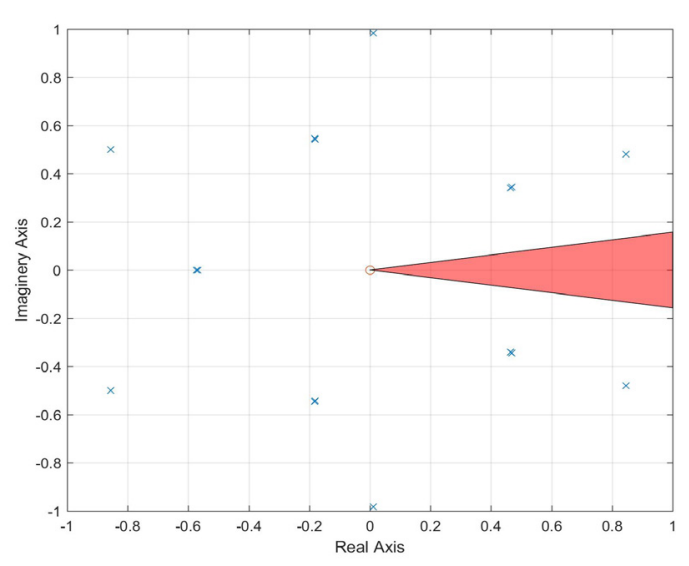

Fig. 7. Stability plot for FOPID controller for link-2

shoot. While in Fig. 9, PID controller has more overshoot and large settling time as compared to FOPID controller. So it can be demonstrated that FOPID controller in cascade with CTC provide better tracking performance as compared to PID controller.

\section{Analysis of Control Effort}

A comparison is given in Fig. 10 and 11 for the control effort required for controlling the robotic arm link-1 and link-2 position using PID and FOPID controllers. The control efforts shown in the Fig. 10 and 11 are for a step response activated at time zero. It is evident from Fig. 10 and 11 that when robotic arm is controlled with FOPID controller it requires remarkably less control effort which is highly desirable criteria for the control of the robotic arm. It is also notable that the initial peak of control effort using the PID scheme is much higher than FOPID as the deriva-

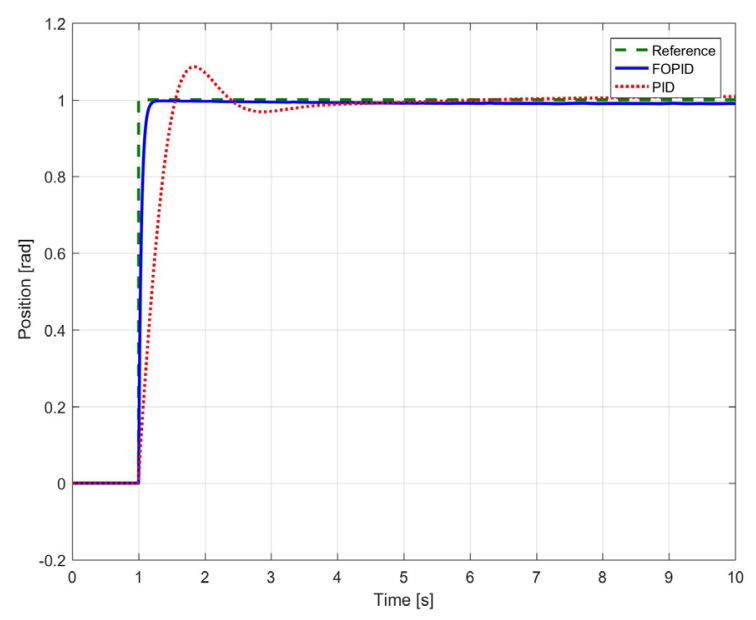

Fig. 9. Tracking performance for robotic arm for link 2 for step input 


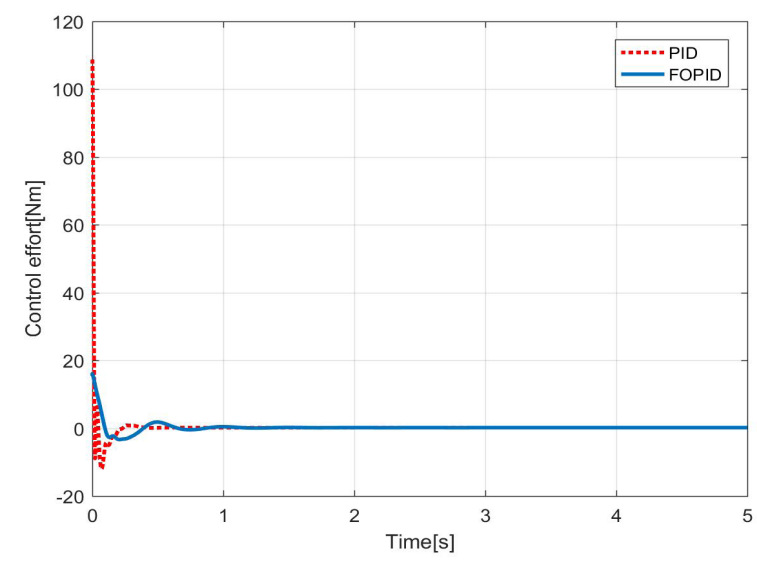

Fig. 10. Control effort comparison for PID and FOPID controller for link 1

tive term of the PID is simulated with a two-steps derivative, while the fractional order derivative is implemented using a digital filter with a finite number of steps based on short-memory principle. So with a two-steps derivative, the initial peak of control effort is higher in case of step input, while with n-steps filter is smoother.

\section{Tracking Performance with Sinusoidal Input}

To check the performance of proposed scheme in finite frequency range, two sinusoidal signals of amplitude $1 \mathrm{~N}$ and frequency $1 \mathrm{~Hz}$ is applied to both links and tracking performance is compared with PID controller. It can be seen from the Fig. 12 and Fig. 13, tracking performance is more improved with the proposed strategy as compared to PID controller.

4. Tracking Performance in the presence of External Disturbance

The tracking performance of nonlinear robotic arm in the existence of disturbance at the control input channel of the plant is shown in Fig. 14 and Fig. 15. In order to show the robustness of the proposed scheme to disturbances, the disturbance has been introduced on the input channels of both links. The disturbance signal, in terms of pulses $0.1 \sin \left(\theta_{1}\right)$ and $0.1 \sin \left(\theta_{2}\right)$ are applied at both of the control input channels of the link-1 and link-2 of the robotic arm respectively. It can be easily seen from the output response in the simulation results that even in the presence of disturbance the proposed scheme i.e., FOPID controller has the ability to better track the system than the classical PID and achieves steady state in less time and also shows better inhibition of oscillations.

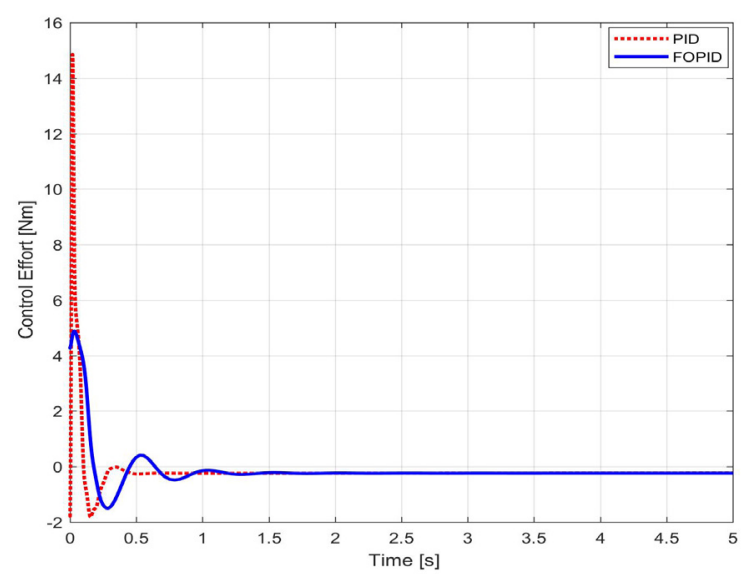

Fig. 11. Control effort comparison for PID and FOPID controller for link 2

\section{CONCLUSION}

This paper offers a novel computed torque controller with the PID controller in the closed loop replaced by FOPID controller design for the accurate and efficient position control of a 2-link robotic arm. The performance of the controller has been evaluated for the transient and steady state performance criteria. The tuning of FOPID controller parameters is done online using the error using Nelder-Mead optimization algorithm and its comparison is shown with the PID controller also tuned with Nelder-Mead. The controllers are validated by applying them on the 2-link nonlinear robotic arm. The effectiveness and improvement can be verified by the simulation results of the FOPID controller tuned using Nelder-Mead technique as compared to NelderMead tuned classical PID controller. Moreover, the robustness of the technique is checked by applying the disturbance. Control effort required for the proposed FOPID controller is also considerably less as compared to classical PID. Performance parameters also indicate that the proposed technique gives quick response and offers better robust stability.

\section{REFERENCES}

1. C. T. Kiang, A. Spowage, and C. K. Yoong, Review of Control and Sensor System of Flexible Manipulator. J. Intell. Robot. Syst. Theory Appl., vol. 77, no. 1, pp. 187-213, 2015.

2. A. Green and J. Z. Sasiadek, Fuzzy and optimal control of a two-link flexible manipulator. Pro- 


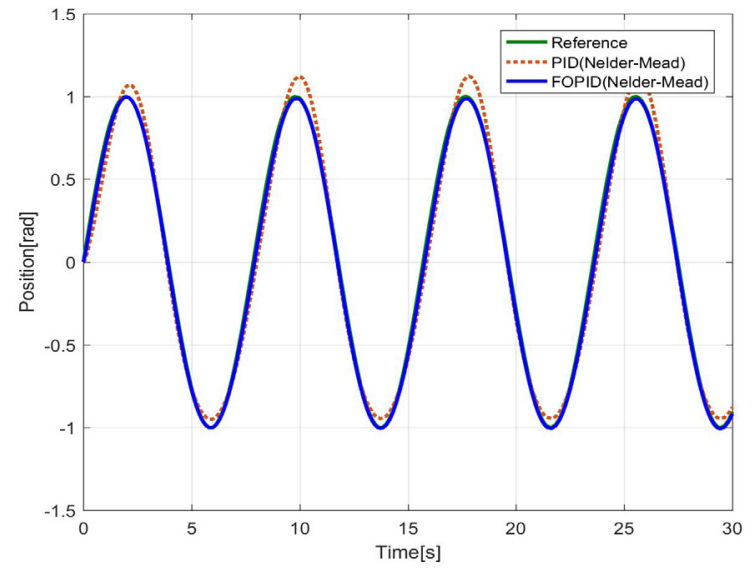

Fig. 12 Robotic arm link-1 tracking performance for sinusoidal input

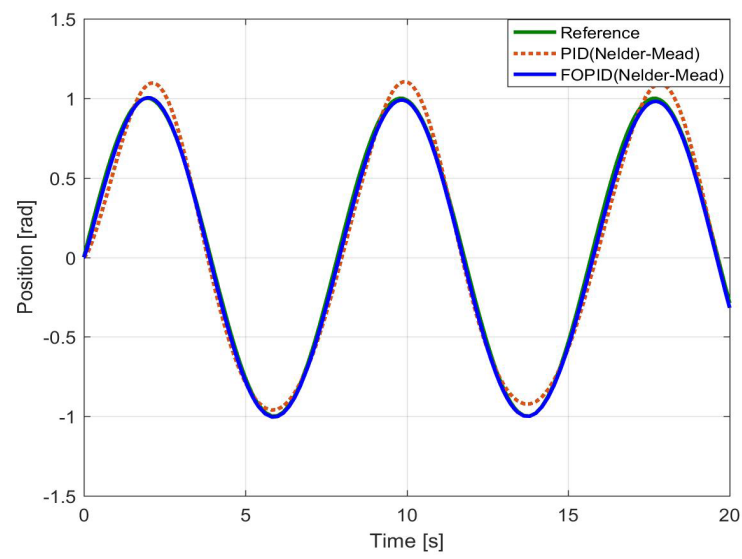

Fig. 14 Tracking performance of robotic arm in the presence of disturbance for link 1

ceedings of IEEE/ASME International Conference on Advanced Intelligent Mechatronics. vol. 2, pp. 1169-1174, 2001

3. U. Gogoi, Model Predictive Control Of A TwoLink Flexible Manipulator. Department of Electrical Engineering, National Institute of Technology, Rourkela, 2015.

4. O. Barambones and V. Etxebarria, Robust neural control for robotic manipulators. Automatica, vol. 8, no. 2, pp. 235-242, 2001.

5. He Wei, Shuzhi Sam Ge, Yanan Li, Effie Chew, and Yee Sien Ng. Neural network control of a rehabilitation robot by state and output feedback. Journal of Intelligent \& Robotic Systems 80, vol.1, pp. 15-31, 2015.

6. Zuo Y, Wang Y, Liu X, Yang SX, Huang L, Wu X, Wang Z., Neural network robust $\mathrm{H} \infty$ tracking control strategy for robot manipulators. Appl. Math. Model., vol. 34, no. 7, pp. 1823-1838, 2010.

7. H. F. Ho, Y. K. Wong, and A. B. Rad, Robust fuzzy tracking control for robotic manipulators. Simul. Model. Pract. Theory, vol. 15, no. 7, pp. 801-816,

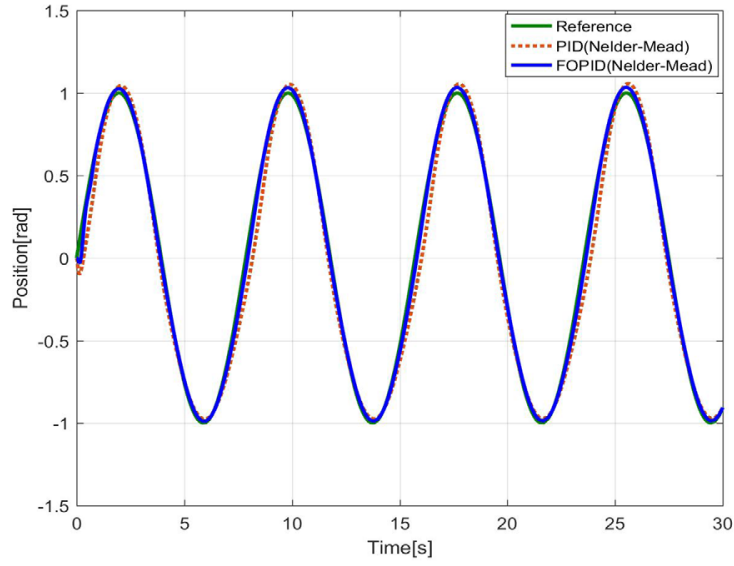

Fig. 13 Robotic arm link-2 tracking performance for sinusoidal input

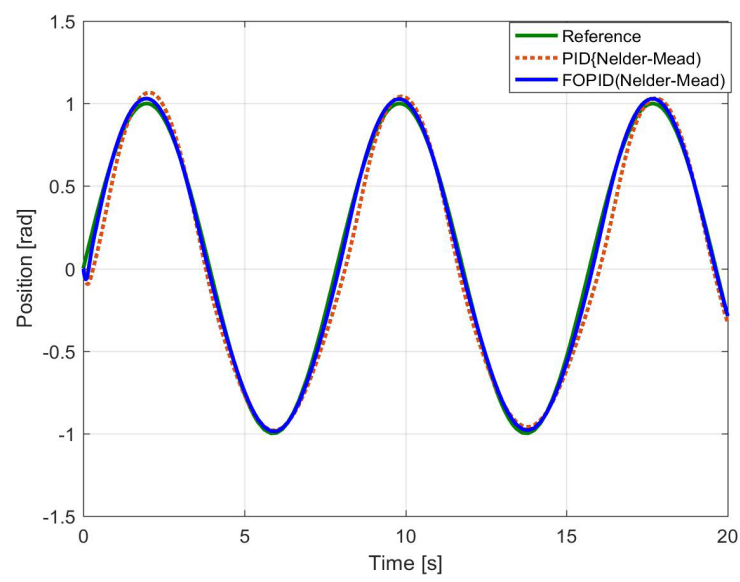

Fig. 15 Tracking performance of robotic arm in the presence of disturbance for link 2

2007.

8. K.-C. Chiou and S.-J. Huang, An adaptive fuzzy controller for robot manipulator. Mechatronics, vol. 15, no. 2, pp. 151-177, 2005.

9. S. H. Hashemipour, A. Ghoreishi, S. M. Mahdavinasab, and M. N. Moghaddasi, PID Controller for Robotic Manipulator Nonlinear Model and Compare with Sliding Mode Controller. Res.J.Recent Sci, vol. 2, no. 11, pp. 50-54, 2013.

10.Z. Song, J. Yi, D. Zhao, and X. Li, A computed torque controller for uncertain robotic manipulator systems: Fuzzy approach. Fuzzy Sets Syst., vol. 154, no. 2, pp. 208-226, 2005.

11. W. Li, X. G. Chang, F. M. Wahl, and J. Farrell, Tracking control of a manipulator under uncertainty by FUZZY PID controller. Fuzzy Sets Syst., vol. 122, no. 1, pp. 125-137, 2001.

12. F. A. T. Al-saedi and A. H. Mohammed, Design and Implementation of PSO-PID Controller for MA2000 Robotic Manipulator. IJCSET, September 2012 .

13. S. Yamamoto, Present status and future needs: the 
view from Japanese industry. Proc. of 4th International Conference on Chemical Process ControlCPC IV, CACHE-AIChE, pp. 1-28, 1991.

14. R. H. Middleton and G. C. Goodwin, Adaptive Computed Torque Control for Rigid Link Manipulators. Syst. Control Lett., vol. 10, no. 1, pp. 9-16, Jan. 1988.

15. M. Uebel, I. Minis, and K. Cleary, Improved computed torque control for industrial robots. Proceedings of IEEE International Conference on Robotics and Automation 1992, pp. 528-533 vol.1. 1992

16. D. Xue, C. Zhao, and Y. Chen, Fractional order PID control of a DC-motor with elastic shaft: a case study. American Control Conference, p. 6 --pp, 2006.

17. S. Karad, S. Chatterji, and P. Suryawanshi, Performance analysis of fractional order PID controller with the conventional PID controller for bioreactor control. Int. J. Sci. Eng. Res., vol. 3, no. 6, pp. 1-6, 2012.

18. N. M. F. Ferreira and J. A. T. Machado, Fractionalorder hybrid control of robotic manipulators. Proc. 11th Int. Conf. Adv. Robot., vol. 398, no. 3, pp. 393-398, 2003

19. C. I. Muresan, C. Ionescu, S. Folea, and R. De Keyser, Fractional order control of unstable processes: the magnetic levitation study case. Nonlinear Dyn., vol. 80, no. 4, pp. 1761-1772, 2015.

20. S. Ijaz, M. T. Humayun, L. Yan, and M. F. Mumtaz, Fractional Order Modeling and Control of Twin Rotor Aero Dynamical System using Nelder Mead Optimization. J Electr Eng Technology, vol. 11, no. 6, pp. 1921-1929, 2016.

21. M. A. S. Aboelela, M. F. Ahmed, and H. T. Dorrah, Design of aerospace control systems using fractional PID controller. J. Adv. Res., vol. 3, no. 3, pp. 225-232, 2012.

22. S. K. Mishra and S. Purwar, To design optimally tuned FOPID controller for twin rotor MIMO system. 2014 Students Conference on Engineering and Systems (SCES), pp. 1-6, 2014.

23. M. F. Silva, J. A. T. Machado, and A. M. Lopes, "Fractional order control of a hexapod robot," Nonlinear Dyn., vol. 38, no. 1-4, pp. 417-433, 2004.

24. L. Bruzzone and P. Fanghella, "Fractional-Order Control of a Micrometric Linear Axis," J. Control Sci. Eng., vol. 2013, p. 4, 2013.

25. R. H. Mohammed, Trajectory Tracking Control for Robot Manipulator using Fractional Order-FuzzyPID Controller. International Journal of Computer Applications, vol. 134, no. 15, p. 8887, 2016.

26. R. Sharma, P. Gaur, and A. P. Mittal, Performance analysis of two-degree of freedom fractional order PID controllers for robotic manipulator with payload. ISA Trans., vol. 58, pp. 279-291, 2015.

27. T. J. E. Eng and C. Sci, Fractional PID controllers tuned by evolutionary algorithms for robot trajectory control. Turkish Journal of Electrical Engineering \& Computer Sciences, vol. 20, pp. 1123-1136, 2012.

28. H. Delavari, R. Ghaderi, a N. Ranjbar, S. H. Hosseinnia, and S. Momani, Adaptive Fractional PID Controller for Robot Manipulator. Proc. 4th IFAC Work. Fract. Differ. Its Appl., vol. 2010, pp. 1-7, 2010.

29. Y. Q. Chen, I. Petráš, and D. Xue, Fractional order control - A tutorial. Proc. Am. Control Conf., no. July, pp. 1397-1411, 2009.

30. Lin, Feng. Robust control design: an optimal control approach. Vol. 18. John Wiley \& Sons, 2007.

31. R. Sharma, P. Gaur, and A. P. Mittal, Optimum Design of Fractional-Order Hybrid Fuzzy Logic Controller for a Robotic Manipulator. Arab. J. Sci. Eng., pp. 739-750, 2016.

32. S. Das, S. Saha, S. Das, and A. Gupta, On the Selection of Tuning Methodology of FOPID Controllers for the Control of Higher Order Processes.ISA transactions, 50(3), pp.376-388. 2012

33. B. M. Vinagre, I. Podlubny, A. Hernandez, and V. Feliu, Some approximations of fractional order operators used in control theory and applications. Fract. Calc. Appl. Anal., vol. 3, no. 3, pp. 231-248, 2000.

34. A. Visioli, Practical PID control. Springer Science \& Business Media, 2006.

35. D. Valério and J. S. da Costa, Tuning of fractional PID controllers with Ziegler--Nichols-type rules. Signal Processing, vol. 86, no. 10, pp. 2771-2784, 2006.

36. J. A. Nelder and R. Mead, A simplex method for function minimization. Comput. J., vol. 7, no. 4, pp. 308-313, 1965.

37. C. Luo and B. Yu, Low Dimensional Simplex Evolution--A Hybrid Heuristic for Global Optimization. Eightth ACIS International Conference on Software Engineering, Artificial Intelligence, Networking, and Parallel/Distributed Computing, vol. 2, pp. 470-474, 2017.

38. A. Moraglio and C. G. Johnson, Geometric generalization of the nelder-mead algorithm. European Conference on Evolutionary Computation in Combinatorial Optimization, pp. 190-201, 2010.

39. N. Ishak, M. Tajjudin, H. Ismail, M. H. F. Rahiman, Y. M. Sam, and R. Adnan, PID studies on position tracking control of an electro-hydraulic actuator. Int. J. Control Sci. Eng., vol. 2, no. 5, pp. 120-126, 2012.

40. A. Tepljakov, E. Petlenkov, and J. Belikov, FOMCON: Fractional-order modeling and control toolbox for MATLAB. Proceedings of the 18th International Conference on Mixed Design of Integrated Circuits and Systems (MIXDES), pp. 684-689, 2011.

41. S. Kr and C. E. Manipal, Stability and Performance Analysis of Fractional Order Control Systems. Wseas Transactions on Systems and Control, vol. 9, pp. 438-444, 2014. 